\title{
Rentabilidad de la implementación de paneles fotovoltaicos en relación con el consumo promedio por vivienda en las 4 regiones naturales del Ecuador.
}

Profitability of the implementation of photovoltaic panels in relation to the average consumption per home in the 4 natural regions of Ecuador

David Ricardo Merchán Sacoto. ${ }^{1}$

\begin{abstract}
.
DOI: https://doi.org/10.33262/concienciadigital.v4i3.1762

Introduction. Technological development is an important part of the global framework of our development, in addition to negatively affecting all ecosystems, we also consume a large part of the resources. Therefore, fundamental measures must be taken to counteract this reality. One of these actions is the application of more self-sustainable constructions in current homes, such as photovoltaic panels. Objective. Evaluate the profitability of the application of the photovoltaic panel system with a view to the sustainability of the building in an economic way. Methodology. In the present study, it was based on the elaboration of an Excel book in which the different calculation methods of the sustainability system are applied based on subsequent comparisons of the behavior of the photovoltaic panels, as previously mentioned it was established in 4 different areas of the country that is Costa; Sierra Oriente and Insular, based on the solar radiation of each area. Results. Among the results in the areas where the implementation of photovoltaic energy occurs based on the average consumption per dwelling, it is not economically feasible, given that to recover the initial investment, the useful life of the equipment is exceeded, which is 20 years. which accumulated savings reaches a value of 8,046.36 USD and recovering the investment at 31 years. Conclution. As for the generation of energy in our country, it is at its peak, so it is not necessary to install photovoltaic panels in places where electricity is generated in a sustainable way, except for affordable electricity. However, the effectiveness of implementing these systems lies in areas where they cannot be accessed.
\end{abstract}

\footnotetext{
${ }^{1}$ Universidad Católica de Cuenca, Maestría en Construcción con Mención en Gestión de Construcción Sostenible, Azuay, Ecuador, david.merchan@est.ucacue.edu.ec, https://orcid.org/0000-0002-4871-004X
} 
Keywords: sustainable building, energy, construction, solar heaters; photovoltaic panels.

\section{Resumen.}

Introducción. El desarrollo tecnológico es parte importante del marco global de nuestro desarrollo, además de afectar negativamente a todos los ecosistemas, también consumimos gran parte de los recursos, Por tanto, se deben tomar medidas fundamentales para contrarrestar esta realidad. Una de estas acciones es la aplicación de construcciones más autosustentables en las viviendas actuales, tales como son los paneles fotovoltaicos. Objetivo. Evaluar la rentabilidad de la aplicación del sistema de paneles fotovoltaicos con miras a la sostenibilidad del edificio de manera económica. Metodología. En el presente estudio, se basó en la elaboración de un libro de Excel en el que se aplica los diferentes métodos de cálculo del sistema de sostenibilidad en base a posteriores comparaciones del comportamiento de los paneles fotovoltaicos, como antes mencionado se estableció en 4 diferentes zonas del país que es Costa; Sierra Oriente e Insular, en base a la radiación solar de cada zona. Resultados. Entre los resultados en las zonas donde se da la implementación de energía fotovoltaica en base al consumo promedio por vivienda no es factible económicamente, dado que para recuperar la inversión inicial se supera el tiempo de vida útil de los equipos, que es de 20 años el cual el ahorro acumulado llega a un valor de 8046,36 USD y recuperando la inversión a los 31 Años. Conclusión. En cuanto a la generación de energía en nuestro país, está en su apogeo, por lo que no es necesario instalar paneles fotovoltaicos en lugares donde se genera electricidad de forma sostenible, salvo electricidad asequible. Sin embargo, la efectividad de implementar estos sistemas radica en áreas donde no se puede acceder a ellos.

Palabras claves: edificio sustentable; energía, construcción; calentadores solares; paneles fotovoltaicos.

\section{Introducción.}

Desde la invención de las herramientas de piedra, la invención de la rueda, el control del fuego hasta toda la existencia de la humanidad, incluso en la tecnología, la arquitectura y las innumerables industrias existentes de nuestro tiempo, los logros de la humanidad alguna vez fueron inimaginables. hoy es más obvio que nunca, como la contaminación excesiva, trae consigo el consumo acelerado de recursos, el cambio climático, el deshielo y el aumento del nivel del mar, temperaturas extremas y muchas otras consecuencias. La Asamblea General en 1987 el Informe "Nuestro Futuro Común", aunque es más conocido como Informe Brundtland (IB), afirma (capítulo 2) que los países No-OCDE no pueden desarrollarse con el modelo de la zona OCDE, debido a la escasez de recursos naturales (especialmente "de la energía, de los materiales, del agua y de tierras"), estos límites "se manifestarán como costes crecientes y rendimientos decrecientes, y no como una pérdida repentina de una base de recursos", por ello el IB urge en el sumario a transformar el modelo económico: "Somos unánimes en la convicción de que la seguridad, el bienestar y la misma supervivencia del planeta dependen de esos cambios ya", que deben producirse "en los viejos enfoque del desarrollo y la protección del medio ambiente" 
(Bermejo Gomez De Segura, 2014). En la $21^{\text {a }}$ Conferencia en París de 2015, alcanzaron un acuerdo histórico con el objetivo de combatir el cambio climático y acelerar e intensificar las acciones y las inversiones necesarias para un futuro sostenible con bajas emisiones de carbono (Organización de las Naciones Unidas, 2011). El desarrollo sostenible es el desarrollo con futuro que garantiza la satisfacción de las necesidades de las generaciones actuales y futuras sin comprometer los recursos naturales y humanos, aplicando tecnologías para disminuir la producción de agentes contaminantes (Fernández \& Gutiérrez, 2013).

Por otro lado, si no se considera de antemano el concepto de desarrollo sostenible, es imposible resolver el problema de la construcción sostenible, que es un desarrollo que satisface las necesidades actuales sin comprometer las capacidades ambientales y no tendrá un impacto negativo en las necesidades de las generaciones futuras. Por lo tanto, las reflexiones aquí presentadas deben comenzar primero con el significado global del desarrollo sostenible, desde su significado original hasta los planes, acuerdos, compromisos y declaraciones producidos por las distintas reuniones del organismo de Naciones Unidas desde entonces. 80, 90 y fecha, anteriormente explicados. Posteriormente, el acuerdo del "Programa Hábitat" incluía los siguientes derechos: Obtener una vivienda adecuada, las consideraciones conceptuales de la construcción sustentable y los principios de su aplicación e implementación de los principios arquitectónicos (Delia \& López, 2010).

Con el uso de combustibles fósiles y En Ecuador, representará alrededor del 50\% de la generación total de electricidad para 2013, y la energía renovable está Se consideran sostenibles porque sus características les permiten satisfacer la demanda energética sin reducir futuro (F \& Espinoza, 2016). Sin embargo, estamos apenas iniciando una campaña de uso de energías sustentable dentro de nuestro país, esto debido a los grandes incentivos que se realiza a las grandes empresas por parte del gobierno central, mencionados incentivos son subsidios a combustibles fósiles que fortalecen el consumo de los mismos por parte de la población dentro del país.

Sin embargo, en la última década las políticas públicas que se han formulado actualmente abren acortan la brecha para fomentar energías limpias, tales como vehículos exonerados que contaminan menos, crecimiento de la producción de electricidad por la construcción de hidroeléctricas, construcción sustentable, impuestos verdes entre otros. El 82\% de la matriz energética de Ecuador obtuvo Petróleo, como diésel, gasolina y gas licuado de petróleo. Sin embargo, Ecuador es internacionalmente como uno de los países con mayores subsidios al combustible, el mayor número de registros el porcentaje de estos subsidios supera el porcentaje de educación y salud. Subsidio de combustible la demanda anual de Ecuador supera los US \$3.000 millones, lo que equivale al 17\% de la demanda total del país. Presupuesto General del Estado (PGE) (Rivera, 2018).

El derivado del petróleo más subsidiado de Ecuador es el diésel, el precio del producto en el país es aproximadamente el 50\% de su precio en el mercado internacional, también señaló los principales problemas relacionados con estos subsidios. Además del impacto 
ambiental causado por el aumento de los costos presupuestarios y del consumo, el bajo precio de venta de los hidrocarburos en comparación con el mercado internacional también ha provocado el traslado de productos a la frontera, provocando enormes pérdidas económicas para Ecuador (Rivera, 2018).

La implementación de impuestos justos sociales y ambientales evitará el abuso de los recursos naturales, los cuales alentará la recolección de recursos que pueden usarse para financiar programas de los mismos y promover el desarrollo de tecnologías limpias (Rivera, 2018).

Un programa de implementación de energías limpias es la construcción el cual hace referencia a la gran importancia para el crecimiento de largo plazo. Sin embargo, hay una gran dependencia de recursos naturales para la industria, el comercio y la construcción lo que demanda grandes cantidades de energía fósil, además de la notable explosión demográfica global (Antero \& Ramírez, 2014).

La demanda de energía se distribuye por sectores, encabezando el consumo energético mundial el cual corresponde al industrial, seguido del transporte y el residencial principalmente con participaciones del 28,9\%, 28,8\% y 21,9\% respectivamente, debido a esto el abastecimiento de demanda energética mundial proporcionada por fuentes primarias no renovables advierte un descenso para 2040, constituyendo el $75 \%$ de la demanda comparado al 81\% de años anteriores (Lady \& David, 2019). Por todo ello, es vital que la industria de la construcción tome medidas drásticas para reducir sus impactos con el fin de reducir su huella ecológica. Si bien algunos países han dado el primer paso para implementar medidas que requieren la instalación de sistemas de protección ambiental, sistemas de generación de energía limpia y otras medidas. Sin embargo, esto no es suficiente porque solo representan una pequeña parte de la energía consumida por estos países y están en el consumo global de energía. En países en vías de desarrollo como Ecuador, estas normas son relativamente nuevas y casi no tienen aplicación, por lo que además de la obvia necesidad de cambiar el pensamiento, la educación y la construcción su socialización también es fundamental y su aplicación debe ser obligatoria. La Arquitectura Sostenible reflexiona sobre el impacto ambiental de todos los procesos implicados en una vivienda desde los materiales de fabricación que no produzca desechos tóxicos y no consuma mucha energía, las técnicas de construcción que supongan un mínimo deterioro ambiental, la ubicación de la vivienda y su impacto con el entorno; el consumo de energía de la misma y su impacto continuando con el reciclado de los materiales cuando la casa ha cumplido su función (Velepucha, 2014). La generación de energía eléctrica a base de fuentes solares y eólicas se ha facultado en el Ecuador a través del organismo responsable de regular el mercado eléctrico, como alternativa para reducir las emisiones de $\mathrm{CO} 2$ y fomentar el uso de fuentes limpias para la generación de corriente eléctrica, cumpliendo con los parámetros técnicos que le permitan evacuar energía eléctrica al Sistema Nacional Interconectado (Moncayo Picerno, 2016).

Sin embargo, en Ecuador se han realizado varios proyectos a pequeña escala, particularmente en aplicaciones fotovoltaicas las mismas que piden apoyo por parte del 
estado, tanto en la investigación como desarrollo y su aplicación, por eso el instituto nacional de eficiencia energética y energías renovables (INER, 2016), está dando los primeros pasos para evaluar el recurso solar y utilizar esta información como base para futuros proyectos (Rayas, 2016).

Un ejemplo de construcción sustentable es la instalación de paneles fotovoltaicos, pues debido a los altos niveles de radiación de Ecuador (aproximadamente $4.2 \mathrm{kWhm}$ ), es rentable considerar el uso de recursos solares, lo que abre una realidad para el sector productivo. La energía anima activamente a todos los ciudadanos a participar en la protección del medio ambiente, porque la energía del sol no produce residuos tóxicos ni gases de efecto invernadero y permite que sus beneficios económicos se distribuyan democráticamente entre toda la población. Como en muchos países el desarrollo en Ecuador la popularización y el desarrollo de sistemas de energía fotovoltaica y/o eólica han sido conectados a la red aún está en su infancia (Velasco, n.d.).

El desarrollo energético de Ecuador a través de recursos renovables aumentará el nivel de producción de energía y aumentará la proporción de viviendas que brindan servicios de electricidad. Además, la expansión, desarrollo e innovación tecnológica del sistema de generación de energía brindará una fuente de trabajo para la comunidad y traerá beneficios económicos al Ecuador.

Este articulo pretende mostrar la capacidad energética que se podría obtener a través de un sistema fotovoltaico para abastecer a una edificación en el sur de la ciudad de Cuenca.

\section{Módulos fotovoltaicos.}

Los módulos fotovoltaicos o también llamados colectores solares fotovoltaicos están formados por un conjunto de celdas fotovoltaicas interconectadas entre ellas en modo de serie o paralelo, dependiendo de este tipo de conexiones es que se pueden obtener paneles solares de diferentes tensión y corrientes generadas (Minotta Marin, 2017), Los paneles fotovoltaicos son los encargados de convertir directamente la energía de la radiación solar en energía eléctrica.

Las fabricaciones de estos paneles solares son con materiales como el silicio es uno de los más usados, los cuales derivan de paneles de silicio monocristalinos y policristalinos, lo cual hace altamente aplicable su uso. (Laborde, 2016)

En las últimas dos décadas los líderes académicos y de opinión pública han publicado artículos interesantes y técnicamente valiosos sobre la aplicación de componentes generadores de energía limpia a las viviendas. La existencia de toda esta información puede basarse en artículos científicos. La experiencia y los trabajos académicos han inspirado el análisis de rentabilidad actual del uso de elementos que generan energía limpia en Ecuador.

Esta investigación tiene un carácter documental experimental y se apoya en una extensa consulta bibliográfica sobre el mismo y/o temas relacionados en el medio. Siguiendo la misma lógica, el experimento se llevó a cabo en cuatro áreas de la región Sierra, Costa y 
Zona Amazónica e Insular con el fin de determinar en cual zona es rentable la aplicación del módulo fotovoltaico.

\section{Aplicaciones.}

Los fotovoltaicos aprovechan la radiación solar debido a que es una de las fuentes que nunca se va acabar, además como ya se ha mencionado con anterioridad es amigable con el ambiente y muy silenciosa para la producción de electricidad (Pasqualino, 2015), por lo que se puede aplicar aprovechando de dos formas:

- Instalaciones aisladas de la red eléctrica: con sistemas fotovoltaicos autónomos que nos sirve para electrificación rural, señalización, bombeo de agua, comunicaciones y más ejemplos como se muestra en el diagrama (Rayas, 2016).

- Instalaciones conectadas a la red eléctrica: centrales fotovoltaicas y edificios conectados a la red (Rayas, 2016).

\section{Posición del módulo fotovoltaico.}

Las posiciones de los módulos fotovoltaicos están especificadas mediante dos coordenadas angulares:

- Angulo acimutal: Está formado por la proyección sobre el plano horizontal perpendicular a la superficie del módulo y el meridiano en esa posición. El grado del valor del módulo es: Sur $0^{\circ}$, Oeste $90^{\circ}$, Norte $180^{\circ}$ y Este $270^{\circ}$ (Farfán, 2015).

- Angulo de elevación: es el que se forma por la superficie del módulo y el plano horizontal. Los grados que se toma en posición horizontal es de $0^{\circ}$ y en forma vertical $90^{\circ}$ (Farfán, 2015).

\section{Funcionamiento de un sistema fotovoltaico}

El funcionamiento de un sistema fotovoltaico es posible gracias al a los paneles solares donde el efecto fotoeléctrico de la energía solar se convierte en energía eléctrica de corriente directa, la cual no se puede utilizar de forma convencional si no es transformada en corriente alterna (Rosas Luna, 2019).

Es aquí donde entra en juego la función del inversor pieza clave del sistema fotovoltaico, ya que es él quien convierte la corriente para que sea compatible con cualquier tipo de instalación. Posteriormente, dependiendo del tipo de sistema fotovoltaico, se pueden tener controladores de cargas que regulan el uso de la energía y un banco de baterías que permita el almacenamiento de la energía; otro elemento importante es el centro de carga, ya que suele ser el punto de conexión o de distribución de la energía fotovoltaica (Cárdenas et al., 2019).

\section{Metodología.}

En el presente estudio, se basó en la elaboración de un libro de Excel en el que se aplica los diferentes métodos de cálculo del sistema de sostenibilidad en base a posteriores 
comparaciones del comportamiento de los paneles fotovoltaicos, como antes mencionado se estableció en 4 diferentes zonas del país que es Costa; Sierra Oriente e Insular, en base a la radiación solar de cada zona.

Con la ayuda de datos presentados en la tabla 1, podemos evaluar el comportamiento de consumo promedio en una vivienda la cual es una de las condiciones para las cuales se puedan proyectar la cantidad de colectores en una vivienda para cada una de las zonas del Ecuador en estudio.

Tabla 1. Promedio de consumo en una vivienda

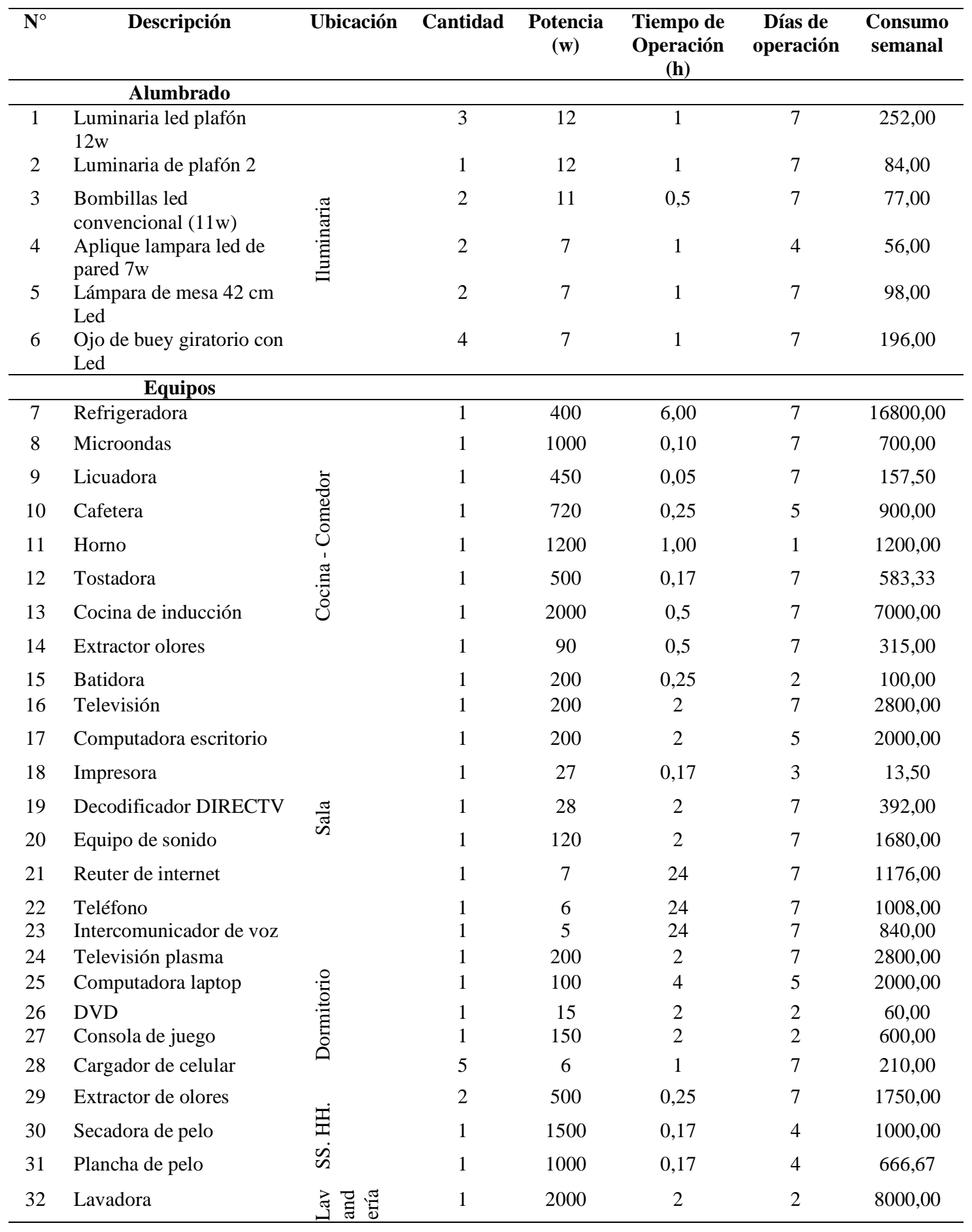




\begin{tabular}{|c|c|c|c|c|c|c|c|}
\hline 33 & Secadora & & 1 & 2000 & 2 & 2 & 8000,00 \\
\hline 34 & Aspiradora & & 1 & 1500 & 1 & 1 & 1500,00 \\
\hline 35 & Plancha & & 1 & 1500 & 1 & 2 & 3000,00 \\
\hline 36 & Bomba & Máquinas & 1 & 745,7 & 0,25 & $\begin{array}{l}3 \\
\text { Total: }\end{array}$ & $\begin{array}{c}559,28 \\
\mathbf{6 8 5 7 4 , 2 7 5}\end{array}$ \\
\hline & & 18425,7 & $\mathrm{~W}$ & & $\mathbf{9 7 9 6 , 3 2 5}$ \\
\hline \multicolumn{3}{|c|}{ Porcentaje de simultaneidad: } & & $50 \%$ & & & \\
\hline \multicolumn{3}{|c|}{ Carga conectada: } & & 9213 & $\mathrm{w}$ & & \\
\hline \multicolumn{3}{|c|}{ Superficie construida: } & & 96,89 & $\mathrm{~m}^{2}$ & & \\
\hline \multicolumn{3}{|c|}{ Parámetro de consumo de energía: } & & 101,11 & Wh/día/m2 & & \\
\hline
\end{tabular}

Fuente: Autoría propia

\section{Radiación solar zona costa.}

En el gráfico 1, indica que la radiación solar media máxima en la ciudad de Manta a una latitud de 0,95 S es 4,39 (KW h / m2), y el valor mínimo de a una latitud de 1,37 S es de 3,31 (KW h / m2) ubicada en NARANJA -Ciudades JIPIJAPA.

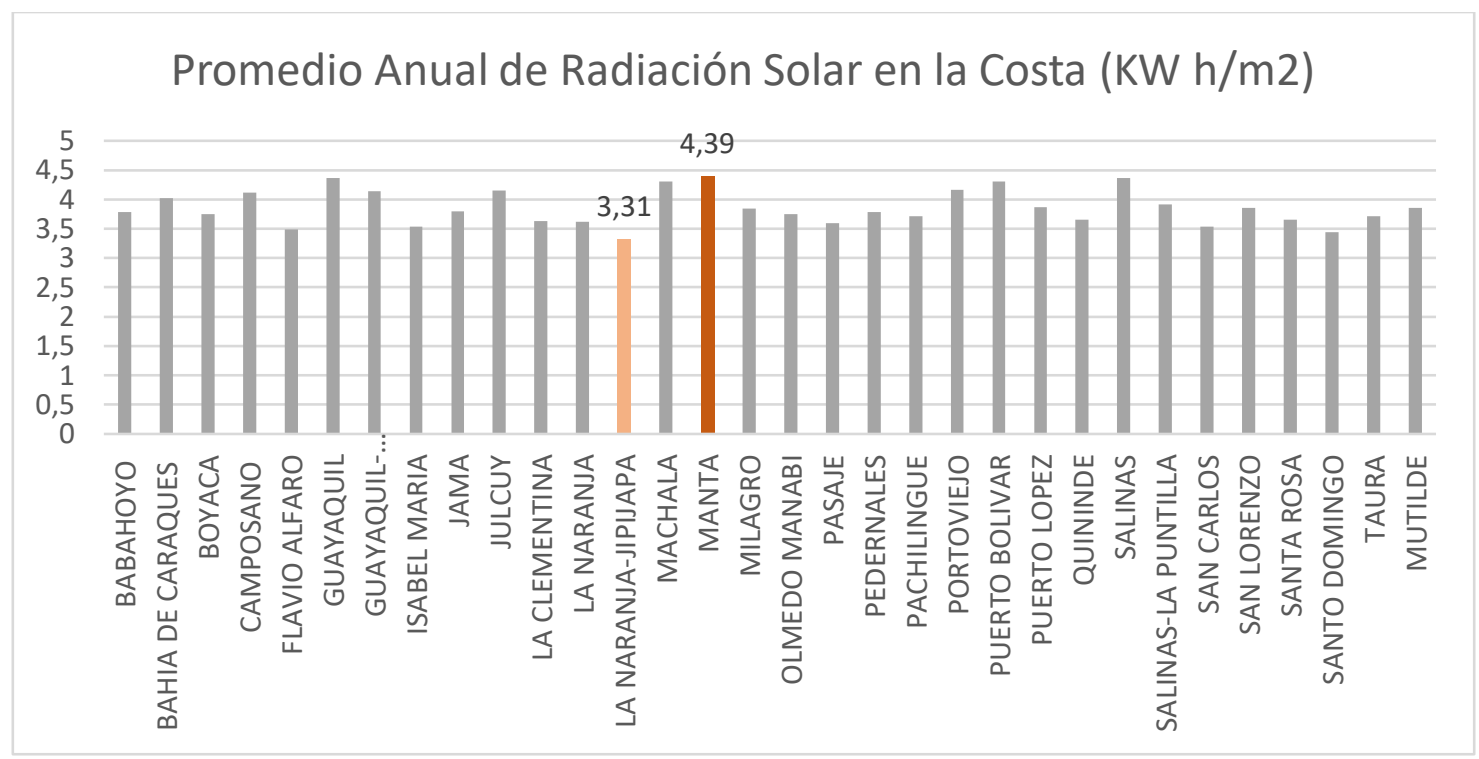

Gráfico 1. Promedio anual de radiación solar en la Costa

Fuente: Autoría propia.

\section{Radiación solar zona sierra.}

La radiación solar media máxima en la ciudad de NAQUITO - QUITO a una latitud de 0,13 S es 4,99 (KW h / m2), y el valor mínimo de a una latitud de 0,95 S es de 3,41 (KW h / m2) ubicada en SAN JUAN - COTOPAXI, como nos demuestra en el gráfico 2. 
Promedio Anual de Radiacion Solar en la Sierra (kw/m2)

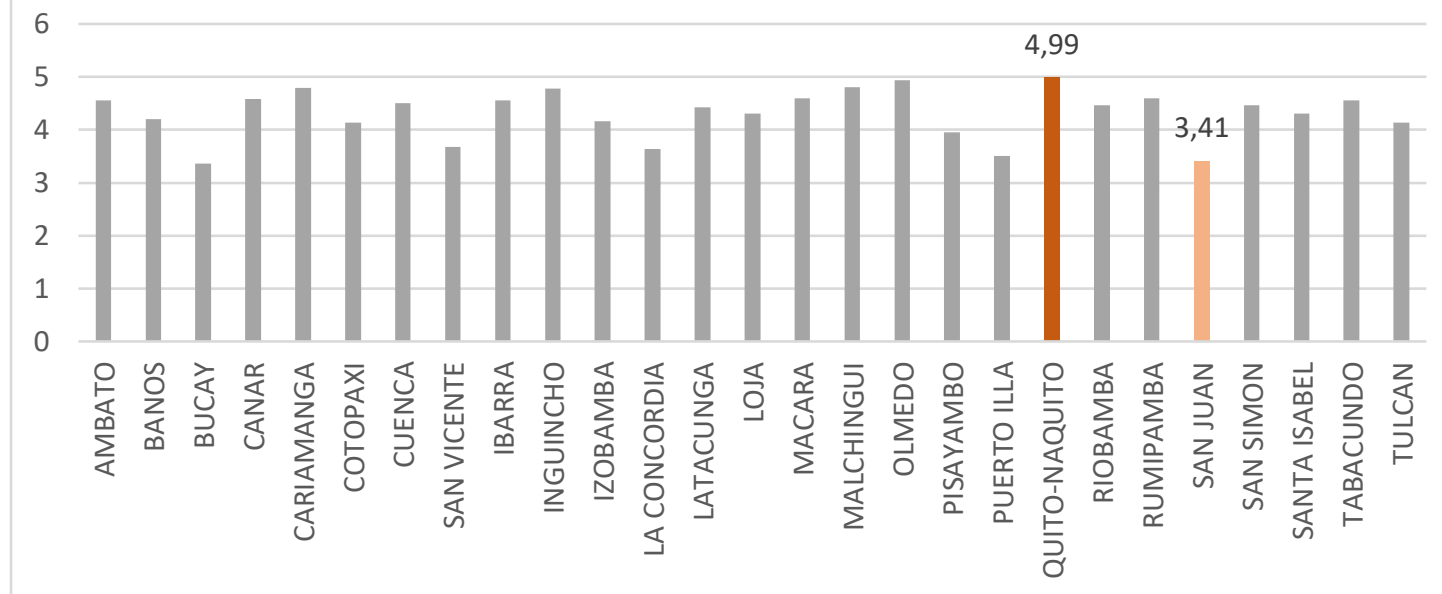

Gráfico 2. Promedio anual de radiación solar en la Sierra

Fuente: Autoría propia

\section{Radiación zona Oriente.}

En el gráfico 3, nos demuestra que el valor máximo de radiación solar promedio es de 4,33 (KW h/m2), en la ciudad de NUEVO ROCAFUERTE - ORELLANA con una latitud de 0,92 S, y la mínima lo tenemos con un dato de 3,77 (KW h/m2), una latitud de $0,95 \mathrm{~S}$ en la ciudad de la SANGAY - MORONA SANTIAGO.

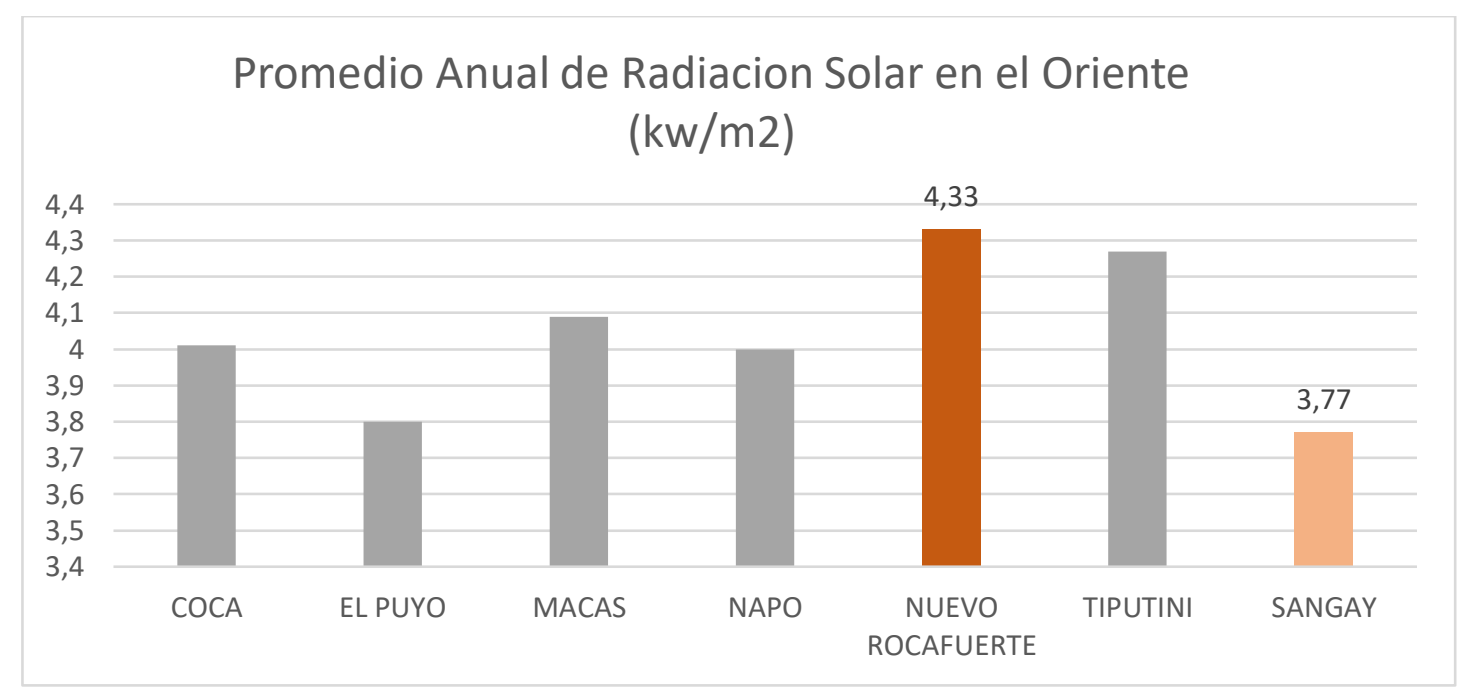

Gráfico 3. Promedio anual de radiación solar en el Oriente

Fuente: Autoría propia.

\section{Radiación zona Insular.}

El gráfico 4, denota que el valor máximo de radiación solar promedio es de 5,31 (KW $\mathrm{h} / \mathrm{m} 2$ ) en la ciudad de PUERTO BAQUERIZO - GALAPAGOS con una latitud de 0,9 $\mathrm{S}$, y la mínima lo tenemos con un dato de 4,41 (KW h/m2) a una latitud de $0,9 \mathrm{~S}$ en la ciudad de la SANTA CRUZ - GALAPAGOS. 
Promedio Anual de Radiacion Solar en la Region Insular (KW h/m2)

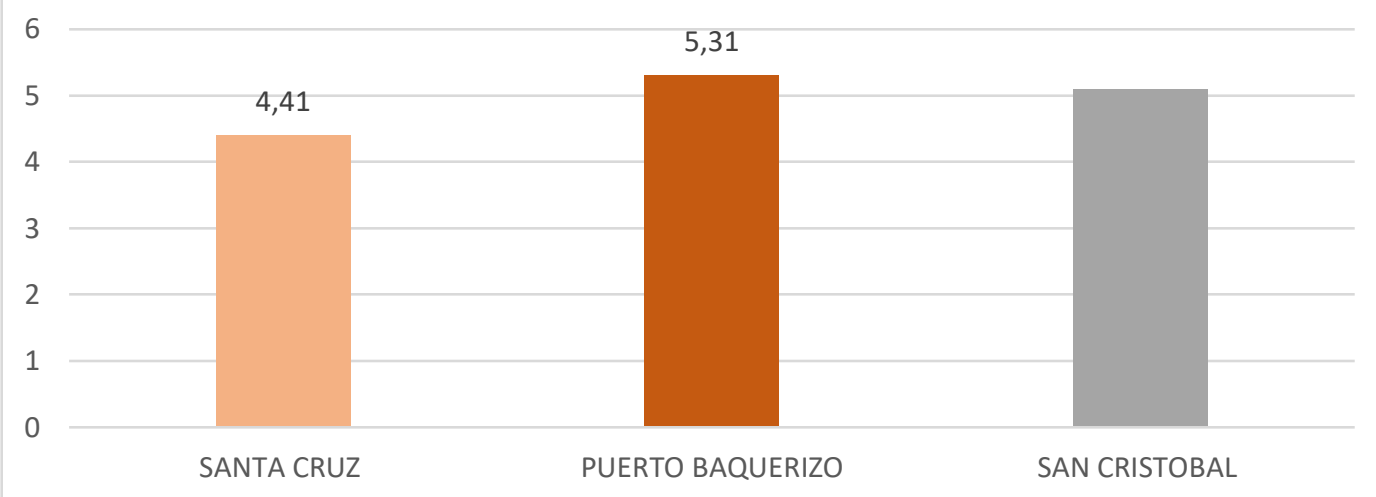

Gráfico 4. Promedio anual de radiación solar en la zona Insular

Fuente: Autoría propia

\section{Cálculo de paneles fotovoltaicos.}

Para establecer el tipo de módulo fotovoltaico utilizado en este caso de estudio, calculamos la potencia (en vatios) de cada electrodoméstico y el tiempo de trabajo del día con el valor de mes más desfavorable como unidad; luego determinamos el mes con las mismas condiciones en Wh. Consumo diario (ET) para posteriormente determinar la profundidad máxima de descarga permitida por el acumulador; a continuación, utilizamos la siguiente fórmula para calcular la energía necesaria:

$$
\begin{gathered}
E=E_{T} I R \\
R=1-\left[\frac{(1-k b-k c-k v) k a N}{p d}\right]-k b-k c-k v
\end{gathered}
$$

De donde:

Siendo R el rendimiento general de la instalación (Cárdenas et al., 2019).

$k_{a}=$ coeficiente de auto descarga de la batería, asumido un valor de 0.003 del fabricante $k_{b}=$ coeficiente de pérdidas de la batería, asumido un valor de 0.05 del fabricante

$k_{c}=$ coeficiente de perdidas del inversor, asumido un valor de 0.2 convertidor de onda senoidal

$k_{v}=$ coeficiente de perdidas varias, asumido un valor de 0.15

$N=$ Número de días de autonomía, calculado un valor de 4.81

$p d=$ Profundidad de descarga, asumido un valor de 0.5

$T=$ Temperatura media ambiente en el más desfavorable, $11.76^{\circ} \mathrm{C}$ 
$R_{a}=$ Radiación solar, se asume el valor de $14.94 \mathrm{MJ} / \mathrm{m}^{2}$.

(Pilco \& Jaramillo, 2008) para determinar en amperes hora, es necesario determinar el voltaje de la instalación lo cual viene dado en la ficha técnica del fabricante, los siguientes datos:

Potencia del módulo $=450 \mathrm{~W}$

Voltaje nominal del módulo $=36 \mathrm{~V}$

Tensión de operación $=72 \mathrm{~V}$

Capacidad de batería $=2040 \mathrm{Ah}$

Voltaje de batería $=2 \mathrm{~V}$

1. Calculamos la capacidad en Amper:

$$
\text { Capacidad en Amper }=\frac{\text { Capacidad en } \text { Watts }}{\text { Voltaje de instalacion }}
$$

2. Luego determinamos la capacidad nominal que es igual a:

$$
\text { Capacidad Nominal }=\frac{\text { Capacidad en Amper }}{p d}
$$

3. Calculamos el número de baterías en paralelo necesarias:

$$
\text { Nro. de Baterias en paralelo }=\frac{\text { Capacidad Nominal }}{\text { Capacidad de la bateria }}
$$

4. Obteniendo que para nuestro caso de estudio es necesario 1 batería en conexión en paralelo, adicional obtenemos el número de baterías en conexión en serie.

$$
\text { Nro.de Baterias en serie }=\frac{\text { Tension de operacion }}{\text { Voltaje de la bateria }}
$$

5. Capacidad instalada:

$$
\text { Capacidad }=(\text { baterias en paralelo })(\text { capacidad de la baetria })
$$

6. En el cual el valor de la capacidad instalada es igual a la capacidad nominal calculada, y posteriormente determinamos el total de baterías en el arreglo.

$$
\text { Total de baterias }=\frac{\text { baterias en } \text { paralelo }}{\text { baterias en serie }}
$$


7. Porcentaje de capacidad de las baterías es igual a:

$$
\% \text { Capacidad }=\left(\frac{\text { Capacidad instalada }}{\text { Capacidad nominal }}-1\right) * 100
$$

En el cual el porcentaje debe ser menor a 10

8. Finalmente determinamos el número de horas de sol pico, en el nuestro caso de estudio es de zona urbana con factor de corrección por inclinación y latitud k=1.05 y por condiciones atmosféricas 1.05 y hora de sol pico igual a:

$$
\text { Hora de sol pico }=1.05 * 1.05 * 0.2788=4
$$

9. Energía a suministrar el panel

$$
E=\frac{E}{0.9}
$$

10. Numero de módulos necesarios:

$$
\text { Numero de modulos }=\frac{\text { Energia a suministrar }}{0.9 * \text { Hora sol pico } * \text { Potencia del modulo }}
$$

$$
\text { Numero de modulos }=10
$$

11. Y la potencia a ser instalada es igual a:

$$
\text { Potencia }=\text { Nro. de modulos } * \text { Potencia del modulo }
$$

\section{Resultados y discusión.}

\section{Paneles Fotovoltaicos.}

Las condiciones iniciales en las que se elaboraron los ensayos para el cálculo serán las mismas que se presentaron en los modelos anteriores; modificando únicamente las zonas geográficas y por lo tanto su nivel de irradiación solar de tal manera que se pueda verificar la zona en la que pueda generar beneficios económicos.

Lo que manifiesta la tabla 2 es que en la región donde más se requieren paneles es en la zona de la Costa, en Santa Elena para ser exactos debido a que la Radiación más baja se da en el mes de JULIO de un valor de $2,79(\mathrm{KWh} / \mathrm{m} 2)$ y donde se requiere de menos paneles fotovoltaicos en la zona Insular en Puerto Baquerizo con un valor de 2,41 y una radiación más baja en el año en el mes de septiembre de 4,68 (KWh/m2). 
Tabla 2. Paneles requeridos para las zonas más optimas y deficientes del Ecuador calculadas con las mismas condiciones en todos los casos. Radiación en kWh/m2

\begin{tabular}{cccccc}
\hline Región & Provincia & Zona & $\begin{array}{c}\text { Radiación } \\
\text { más baja del } \\
\text { año } \\
\left(\mathbf{K W h} / \mathbf{m}^{\mathbf{2}}\right)\end{array}$ & Mes & $\begin{array}{c}\text { Paneles } \\
\text { requeridos }\end{array}$ \\
\hline COSTA & MANABI & MANTA & 4 & Junio & 2,89 \\
COSTA & SANTA ELENA & SALINAS & 2,79 & Julio & 4,04 \\
SIERRA & PICHINCHA & QUITO & 4,53 & Abril & 2,48 \\
SIERRA & AZUAY & CUENCA & 3,56 & Junio & 2,51 \\
ORIENTE & ORELLANA & ROCAFUERTE & 3,7 & Junio & 3,01 \\
ORIENTE & NAPO & NAPO/SAN VICENTE & 3,4 & Septiembre & 3,32 \\
INSULAR & GALAPAGOS & PUERTO BAQUERIZO & 4,68 & Septiembre & 2,41 \\
INSULAR & GALAPAGOS & SANTA CRUZ & 3,5 & Agosto & 3,18 \\
\hline
\end{tabular}

Fuente: Autoría propia.

Al variar los datos de radiación se necesitará un mayor o menos número de módulos fotovoltaicos que sean capaces de abastecer los requerimientos para la carga de los acumuladores.

Por lo tanto, el número de baterías requeridas depende de la carga que se pretenda alimentar; factor constante para todos los diseños que se presentaron en la tabla 1, el resultado es exactamente igual; generando la necesidad de incluir 2 unidades promedio en serie para obtener los $24 \mathrm{~V}$ dc determinados en nuestro diseño y 4 unidades conectadas en paralelo para soportar la capacidad requerida. Entonces, el número total es de 8 baterías de $150 \mathrm{Ah}$.

\section{Cálculo de ahorro.}

Para determinar el cálculo de ahorro se utilizaron los siguientes parámetros que se determinan en la tabla 3, los cuales indican el costo que se requiere para la instalación de un panel fotovoltaico.

Tabla 3. Parámetros iniciales para instalación de paneles fotovoltaicos

\begin{tabular}{ll}
\hline Descripción & Costo \\
Panel Solar & 0,33 USD/W \\
Inversor & 0,34 USD/W \\
Baterías & 1,4 USD/AH \\
Mano De Obra Especializada & $0,1651 \mathrm{USD} / \mathrm{W}$ \\
Estructura & $0,185 \mathrm{USD} / \mathrm{wp}$ \\
Resto Material & $0,185 \mathrm{USD} / \mathrm{wp}$ \\
Utilidad & \\
Costo & $\mathbf{2 , 6 0 5 1 ~ U S D}$ \\
Área De La Vivienda & $96,89 \mathrm{~m} 2$ \\
Costo Por Metro Cuadrado & $600 \mathrm{USD}$ \\
Costo De La Obra & $58134 \mathrm{USD}$ \\
Porcentaje De Instalación Eléctrica & $5 \%$ \\
Costo De La Instalación Convencional & $2906,7 \mathrm{USD}$ \\
Consumo Diarios De Energía & $9,796325 \mathrm{KWD}$ \\
Consumo Mensual & $297,9715521 \mathrm{KWD}$ \\
\hline
\end{tabular}




\begin{tabular}{ll}
\hline Consumo Anual & 3575,658625 KWD \\
Costo Del Kwh & 0,1 USD/KW \\
Costo Mensual Por Consumo De Energía & 29,79715521 USD \\
Costo Anual Por Consumo & 357,5658625 USD \\
Instalación Fotovoltaica & $\mathbf{6 9 6 8 , 7 3 1 9 ~ U S D ~}$ \\
Potencia El Combo Solar Encontrado & 660 \\
Costo Del Combo Solar & 1890 USD \\
Costo Del Combo Solar Por Watt & 2,863636364 USD/W \\
Instalación & 0,17 USD/W \\
Estructura & 0,22 USD/W \\
Canalizaciones, Cableados, Otros Materiales & 0,22 USD/W \\
Potencia Fotovoltaica & $4500 \mathrm{~W}$ \\
Costo Estimado De La Instalación Solar & 15631,36364 USD/W \\
Costo Diferencial= Costo Inst.Solar- Costo Inst Conv & $\mathbf{1 2 7 2 4 , 6 6 3 6 4}$ \\
Costos De Mantenimiento Al 2\% Anual & $2 \%$ \\
Instalación Convencional & 58,134 \\
Mantenimiento De La Instalación Solar & 312,6272727 USD \\
\hline
\end{tabular}

\section{Fuente: Autoría propia.}

El ahorro en porcentaje es de 62,09\% anuales, que representa un valor de 290, 36586 USD anuales, como lo demuestra la siguiente tabla.

Tabla 4. Ahorro en la instalación de paneles fotovoltaicos

\begin{tabular}{|c|c|c|c|c|c|c|c|c|c|c|c|c|c|}
\hline & Ene. & Feb. & Mar & Abr. & May & Jun. & Jul. & Ago. & Sep. & Oct. & Nov. & Dic. & Anual \\
\hline Consumo & 9,79 & 9,79 & 9,79 & 9,796 & 9,79 & 9,79 & 9,79 & 9,79 & 9,79 & 9,79 & 9,79 & 9,79 & \\
\hline $\begin{array}{l}\text { Diario En } \\
\text { Kwh }\end{array}$ & 6325 & 6325 & 6325 & 325 & 6325 & 6325 & 6325 & 6325 & 6325 & 6325 & 6325 & 6325 & \\
\hline $\begin{array}{l}\text { Número De } \\
\text { Días Del Mes }\end{array}$ & 31 & 28 & 31 & 30 & 31 & 30 & 31 & 31 & 30 & 31 & 30 & 31 & \\
\hline $\begin{array}{l}\text { Consumo } \\
\text { Mensual Wh }\end{array}$ & $\begin{array}{l}303, \\
6860 \\
75\end{array}$ & $\begin{array}{l}274, \\
2971\end{array}$ & $\begin{array}{l}303, \\
6860 \\
75\end{array}$ & $\begin{array}{l}293,8 \\
8975\end{array}$ & $\begin{array}{l}303, \\
6860 \\
75\end{array}$ & $\begin{array}{l}293 \\
8897 \\
5\end{array}$ & $\begin{array}{l}303, \\
6860 \\
75\end{array}$ & $\begin{array}{l}303, \\
6860 \\
75\end{array}$ & $\begin{array}{l}293, \\
8897 \\
5\end{array}$ & $\begin{array}{l}303, \\
6860 \\
75\end{array}$ & $\begin{array}{l}293 \\
8897 \\
5\end{array}$ & $\begin{array}{l}303, \\
6860 \\
75\end{array}$ & $\begin{array}{l}3575, \\
65863\end{array}$ \\
\hline $\begin{array}{l}\text { Costo En } \\
\text { Kwh }\end{array}$ & 0,1 & 0,1 & 0,1 & 0,1 & 0,1 & 0,1 & 0,1 & 0,1 & 0,1 & 0,1 & 0,1 & 0,1 & \\
\hline Costo De & 30,3 & 27,4 & 30,3 & 29,38 & 30,3 & 29,3 & 30,3 & 30,3 & 29,3 & 30,3 & 29,3 & 30,3 & \\
\hline Importe & 6860 & 2971 & 6860 & 8975 & 6860 & 8897 & 6860 & 6860 & 8897 & 6860 & 8897 & 6860 & \\
\hline $\begin{array}{l}\text { Consumo En } \\
\text { El Mes }\end{array}$ & 75 & & 75 & & 75 & 5 & 75 & 75 & 5 & 75 & 5 & 75 & \\
\hline $\begin{array}{l}\text { IVA } \\
\text { Cargo Fijo }\end{array}$ & $\begin{array}{l}12 \% \\
5\end{array}$ & $\begin{array}{l}12 \% \\
5\end{array}$ & $\begin{array}{l}12 \% \\
5\end{array}$ & $\begin{array}{l}12 \% \\
5\end{array}$ & $\begin{array}{l}12 \% \\
5\end{array}$ & $\begin{array}{l}12 \% \\
5\end{array}$ & $\begin{array}{l}12 \% \\
5\end{array}$ & $\begin{array}{l}12 \% \\
5\end{array}$ & $\begin{array}{l}12 \% \\
5\end{array}$ & $\begin{array}{l}12 \% \\
5\end{array}$ & $\begin{array}{l}12 \% \\
5\end{array}$ & $\begin{array}{l}12 \% \\
5\end{array}$ & \\
\hline Costo De & 39,6 & 36,3 & 39,6 & 38,51 & 39,6 & 38,5 & 39,6 & 39,6 & 38,5 & 39,6 & 38,5 & 39,6 & 467,6 \\
\hline Consumo De & 1284 & 2127 & 1284 & 5652 & 1284 & 1565 & 1284 & 1284 & 1565 & 1284 & 1565 & 1284 & 73766 \\
\hline $\begin{array}{l}\text { Energía Al } \\
\text { Mes }\end{array}$ & 04 & 52 & 04 & & 04 & 2 & 04 & 04 & 2 & 04 & 2 & 04 & \\
\hline $\begin{array}{l}\text { Potencia } \\
\text { Fotovoltaica }\end{array}$ & 4500 & 4500 & 4500 & 4500 & 4500 & 4500 & 4500 & 4500 & 4500 & 4500 & 4500 & 4500 & \\
\hline $\begin{array}{l}\text { Horas Sol } \\
\text { Pico }\end{array}$ & 3,49 & 3,48 & 3,81 & 3,98 & 4 & 3,92 & 3,97 & 4,09 & 4,06 & 3,87 & 3,83 & 3,56 & \\
\hline $\begin{array}{l}\text { Energía } \\
\text { Generada } \\
\text { Rendimiento }\end{array}$ & $\begin{array}{l}486, \\
855 \\
60 \%\end{array}$ & $\begin{array}{l}438, \\
48 \\
60 \%\end{array}$ & $\begin{array}{l}531 \\
495 \\
60 \%\end{array}$ & 537,3 & 558 & $\begin{array}{l}529, \\
2 \\
60 \%\end{array}$ & $\begin{array}{l}553 \\
815 \\
60 \%\end{array}$ & $\begin{array}{l}570 \\
555 \\
60 \%\end{array}$ & $\begin{array}{l}548, \\
1 \\
60 \%\end{array}$ & $\begin{array}{l}539, \\
865 \\
60 \%\end{array}$ & $\begin{array}{l}517, \\
05 \\
60 \%\end{array}$ & $\begin{array}{l}496, \\
62 \\
60 \%\end{array}$ & $\begin{array}{l}6307, \\
335 \\
60 \%\end{array}$ \\
\hline Energía Útil & $\begin{array}{l}292, \\
11\end{array}$ & $\begin{array}{l}263, \\
09\end{array}$ & $\begin{array}{l}318, \\
90\end{array}$ & $\begin{array}{l}322,3 \\
8\end{array}$ & $\begin{array}{l}334, \\
80\end{array}$ & $\begin{array}{l}317, \\
52\end{array}$ & $\begin{array}{l}332 \\
29\end{array}$ & $\begin{array}{l}342, \\
33\end{array}$ & $\begin{array}{l}328 \\
86\end{array}$ & $\begin{array}{l}323, \\
92\end{array}$ & $\begin{array}{l}310, \\
23\end{array}$ & $\begin{array}{l}297, \\
97\end{array}$ & $\begin{array}{l}3784, \\
40\end{array}$ \\
\hline $\begin{array}{l}\text { Porcentaje De } \\
\text { Sustitución \% }\end{array}$ & 100 & 100 & 100 & 100 & 100 & 100 & 100 & 100 & 100 & 100 & 100 & 100 & \\
\hline $\begin{array}{l}\text { Déficit En } \\
\text { Kwh }\end{array}$ & 0 & 0 & 0 & 0 & 0 & 0 & 0 & 0 & 0 & 0 & 0 & 0 & \\
\hline $\begin{array}{l}\text { Importe Por } \\
\text { Consumo De } \\
\text { Energía }\end{array}$ & 0 & 0 & 0 & 0 & 0 & 0 & 0 & 0 & 0 & 0 & 0 & 0 & \\
\hline
\end{tabular}




\begin{tabular}{|c|c|c|c|c|c|c|c|c|c|c|c|c|c|}
\hline $\begin{array}{l}\text { Cargo Por } \\
\text { Déficit De } \\
\text { Energía Y } \\
\text { Conexión A } \\
\text { Red }\end{array}$ & 5,6 & 5,6 & 5,6 & 5,6 & 5,6 & 5,6 & 5,6 & 5,6 & 5,6 & 5,6 & 5,6 & 5,6 & 67,2 \\
\hline Ahorro & $\begin{array}{l}24,7 \\
6860 \\
75\end{array}$ & $\begin{array}{l}21,8 \\
2971\end{array}$ & $\begin{array}{l}24,7 \\
6860 \\
75\end{array}$ & $\begin{array}{l}23,78 \\
8975\end{array}$ & $\begin{array}{l}24,7 \\
6860 \\
75\end{array}$ & $\begin{array}{l}23,7 \\
8897 \\
5\end{array}$ & $\begin{array}{l}24,7 \\
6860 \\
75\end{array}$ & $\begin{array}{l}24,7 \\
6860 \\
75\end{array}$ & $\begin{array}{l}23,7 \\
8897 \\
5\end{array}$ & $\begin{array}{l}24,7 \\
6860 \\
75\end{array}$ & $\begin{array}{l}23,7 \\
8897 \\
5\end{array}$ & $\begin{array}{l}24,7 \\
6860 \\
75 \\
\text { Porc } \\
\text { entaj } \\
\text { e De } \\
\text { Ahor } \\
\text { ro }\end{array}$ & $\begin{array}{l}290,3 \\
65863 \\
62,09 \\
\%\end{array}$ \\
\hline $\begin{array}{l}\text { Costo dife } \\
\text { Mantenim }\end{array}$ & $\begin{array}{l}\text { ial } \\
\text { difer }\end{array}$ & cial & & & & $\begin{array}{l}1272 \\
254,\end{array}$ & $\begin{array}{l}, 66364 \\
932727\end{array}$ & & & & & & \\
\hline $\begin{array}{l}\text { Ahorro } \\
\mathrm{t}=\end{array}$ & & & & & & $\begin{array}{l}290, \\
43,8\end{array}$ & $\begin{array}{l}658625 \\
286377\end{array}$ & & & & & & \\
\hline $\begin{array}{l}\mathrm{i}=\text { Tasa in } \\
\mathrm{c}=\text { increm } \\
\mathrm{e}=\text { interés } \\
\text { Interés bal }\end{array}$ & $\begin{array}{l}\text { ión }= \\
\text { en el } \\
\text { ancierc } \\
\text { o }\end{array}$ & $\begin{array}{l}\text { osto } d \\
\text { ara } p\end{array}$ & $\begin{array}{l}\text { los co } \\
\text { ueño }\end{array}$ & $\begin{array}{l}\text { bustib } \\
\text { pital }=\end{array}$ & & & $\begin{array}{r}0,0017 \\
5 \% \\
4,90 \% \\
9 \%\end{array}$ & & & & & & \\
\hline
\end{tabular}

Fuente: Autoría propia

Con la misma lógica esto se aplica al cuadro de resultados de ahorros por años en la cual se aplica en la siguiente gráfica donde los valores más representativos se encuentran marcados. En las zonas donde se da la implementación de energía fotovoltaica en base al consumo promedio por vivienda no es factible económicamente, dado que para recuperar la inversión inicial se supera el tiempo de vida útil de los equipos, que es de 20 años el cual el ahorro acumulado llega a un valor de 8046,36 USD y recuperando la inversión a los 31 Años.

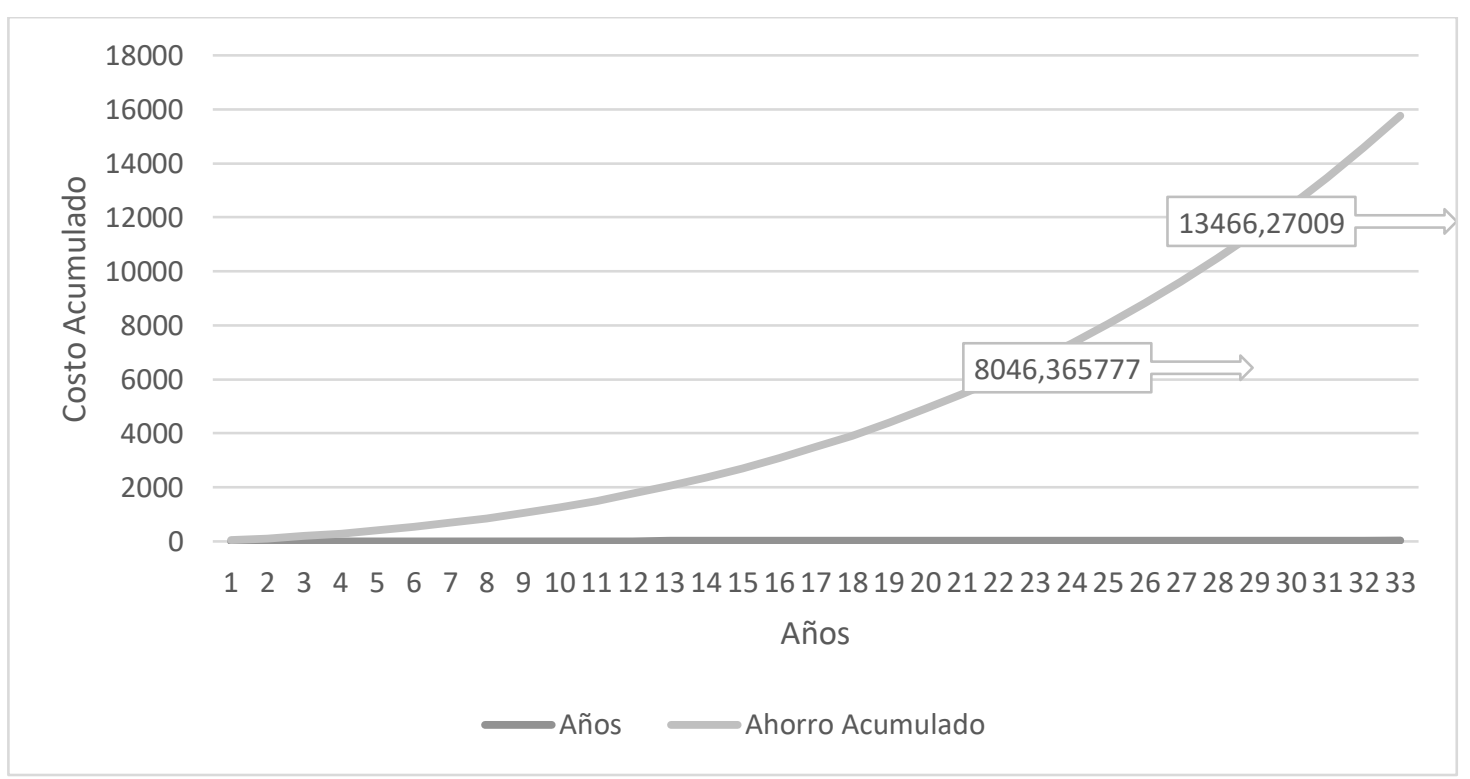

Gráfico 5. Ahorro acumulado vs años

Fuente: Autoría propia.

\section{Conclusión.}

- En el Ecuador se está implementando acciones eco-sustentables como es en el caso de la eco- construcción, que va desarrollándose de manera exponencial en el 
país más de ser un resultado de conciencia ambiental implantada por medio de infinitas fuentes de información que nos inculcan a la educación ambiental, por lo cual es más económico y eficiente que algunos combustibles fósiles. Sin embargo, los subsidios que se da a estos en el país impiden que la disminución de la huella de carbono se ejecute de manera macro; los costos para implementar equipos y sistemas generadores de energía limpia son muy elevados por el momento. Es importante implementar cambios sistemáticos que contribuyan al desarrollo sustentable de la edificación y que satisfagan las necesidades del sector mediante la sensibilización y comprensión del sector energético ambientalmente amigable.

- Utilizando eficazmente la energía de los materiales para el desarrollo de estructuras fotovoltaicas e implementando un diseño utilizado por el sol pasivoactivo, basado en las variables de la geometría solar permiten realizar construcciones que pueden alcanzar valor calorífico sin que exista la necesidad de implementar procedimientos adicionales. Sin embargo, no resulta económicamente factible en el medio local, dado que para recuperar la inversión inicial se supera el tiempo de vida útil de los equipos. Aparte de que existe una extensa infraestructura para generación de energías limpias tales como son las centrales hidroeléctricas que se encuentran a lo largo del país generando un total de 7146 MW, según la Corporación Eléctrica del Ecuador CELEC, por lo tanto, no se recomienda la instalación de estos sistemas en lugares donde la red central de electricidad es constante y sustentable. Por otro lado, la ventaja más clara en la implementación de métodos fotovoltaicos para viviendas unifamiliares, es únicamente en sectores en el cual su ubicación sea complicada o inaccesible para la red pública y/o transporte.

- Finalmente concluimos que con respecto a la generación eléctrica en nuestro país se encuentra en su pico, por lo tanto, no existe la necesidad de implementar paneles fotovoltaicos en lugares donde la electricidad es generada de manera sustentable y además de ser muy asequible. Sin embargo, la efectividad de la implementación de estos sistemas recae en zonas donde su ubicación sea inaccesible.

\section{Agradecimientos.}

El presente artículo es parte del trabajo de investigación y titulación del Programa de Maestría en Construcción con Mención en Administración de la construcción Sustentable de la Universidad Católica de Cuenca, por ello agradecemos a todos y cada uno de los instructores por los conocimientos e información brindados parala elaboración del trabajo.

\section{Referencias.}

Antero, J., \& Ramírez, V. (2014). Evolución De Las Teorías De Explotación De Recursos Naturales: Hacia La Creación De Una Nueva Ética Mundial. Luna Azul, 39, 291313. 
Bermejo Gomez De Segura. (2014). Del desarrollo sostenible según Brundtland a la sostenibilidad como biomimesis. In Del desarrollo Sostenible según Brundtlant a la sostenibilidad como biomimesis.

Cárdenas, V., Álvarez, R., \& González, M. (2019). Inversores inteligentes en sistemas de energía solar fotovoltaica. Journal of Chemical Information and Modeling, 53(9), $24-29$.

Delia, M. A., \& López, C. (2010). Caso : la vivienda de interés social en la ciudad de Mexicali, Baja California. México. .”.

F, L. U., \& Espinoza, J. L. (2016). Energia solar en el Ecuador (Issue January).

Fernández, L., \& Gutiérrez, M. (2013). Bienestar social, económico y ambiental para las presentes y futuras generaciones. Informacion Tecnologica, 24(2), 121-130. https://doi.org/10.4067/S0718-07642013000200013

Lady, A., \& David, A. (2019). “Análisis De Escenarios De Eficiencia Energética En El Sector Residencial Del Cantón Cuenca Utilizando El Modelo Leap.” 144.

Moncayo Picerno, S. A. (2016). Universidad Politécnica Salesiana Sede Quito. Tesis, 1100.

Organización de las Naciones Unidas. (2011). Cambio climático.

Pilco, D., \& Jaramillo, J. (2008). Sistemas fotovoltaicos para iluminación: paneles fotovoltaicos. Universidad Técnica Particular Loja, O(Profesional en formación, Universidad Técnica Particular de Loja \#2Docente, Universidad Técnica Particular de Loja), 1-4.

Rivera, P. Á. (2018). SUBSIDIOS A LOS COMBUSTIBLES FÓSILES EN ECUADOR : 28(1), 87-106.

Velasco, G. F. (n.d.). Generación solar fotovoltaica dentro del esquema de generación distribuida para la provincia de Imbabura.

Velepucha, D. (2014). Propuesta Sustentable, Aplicada a Una Vivienda Saludable.

\section{LCiencia}




\section{PARA CITAR EL ARTÍCULO INDEXADO.}

Merchán Sacoto, D. R. (2021). Rentabilidad de la implementación de paneles fotovoltaicos en relación con el consumo promedio por vivienda en las 4 regiones $\begin{array}{llll}\text { naturales del } & \text { Ecuador. } & \text { ConcienciaDigital, } & \text { 4(3), }\end{array}$ https://doi.org/10.33262/concienciadigital.v4i3.1762

\section{¿Ciencia}

El artículo que se publica es de exclusiva responsabilidad de los autores y no necesariamente reflejan el pensamiento de la Revista Conciencia Digital.

El artículo queda en propiedad de la revista y, por tanto, su publicación parcial y/o total en otro medio tiene que ser autorizado por el director de la Revista Conciencia Digital.

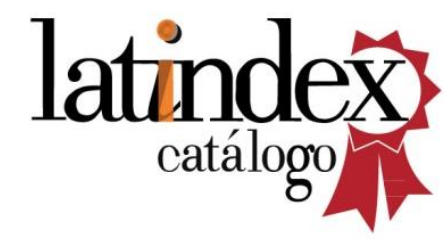

